\title{
An experimental model of the evaporative cooling system of a single powerful LED
}

\author{
Evgeny Shatskiy
}

Kutateladze Institute of Thermophysics SB RAS, 630090, 1 Lavrentyev Ave., Novosibirsk, Russia Novosibirsk state University, 630090, 2 Pirogova Str., Novosibirsk, Russia

\begin{abstract}
An experimental model of the evaporative cooling system of a single powerful LED with a natural circulation of the coolant capable of removing a heat flux density of more than $1 \mathrm{~kW} / \mathrm{cm} 2$ is created. It is shown that on the finned surfaces the overheating relative to the saturation temperature in comparison with a smooth surface decreases up to three times for the heater with a diameter of $5 \mathrm{~mm}$. There is up to two times increase in heat transfer coefficient on finned surfaces as compared to the smooth ones. For finned surfaces on the heater with a diameter of $1 \mathrm{~mm}$ the surface overheating relative to the saturation temperature decreases in four times. More than three times increase is observed for the heat transfer coefficient on finned surfaces as compared to the smooth ones.
\end{abstract}

\section{Introduction}

The future of a number of sectors of the economy is linked with the technology of solidstate light sources and one of the main characteristics of uptime is the removal of heat. Study of the evaporation mechanism on the surfaces of small size was started in [1]. The main objective was to study the temperature fields of the heating surface and liquid, and to study conditions for the formation and existence of a thin film of liquid at the base of the vapor bubble. In this work, as in the follow-up, dedicated to the study of a single center of vaporization [2], the heat flux limited with value at which there is a steady generation of a single center of evaporation, that is relatively modest values. A review of studies on heat transfer during vaporization at the surface of the small size is contained in [3].

Cooling of heat-emitting surfaces in different technological applications is an important problem, which can be solved by such processes as film flows $[4,5]$, etc. In our days the problem of creating an effective compact cooling system for powerful LEDs whose efficiency and durability are highly dependent on the efficiency of the power removal remains unsolved.

Study of evaporation mechanism on the surfaces of small size (SSS) was started in [6]. Heat transfer at boiling of dielectric liquids on the heaters of small size in the sunk vertical channels was studied in [7]. A review of works on heat transfer at evaporation on SSS may be found in [3]. In the literature, there is a dependencies for the critical heat flux density for each type of SSS, having the general form:

$$
q_{c r} / q_{c r o o}=k\left(d_{s} / l_{\sigma}\right)^{n}
$$


but differing in correlation multipliers; and $\mathrm{d}_{\mathrm{s}}$ is the diameter of the heating surface. Here

$$
q_{c r \infty}=0.13 r_{l v} \rho_{v}\left(\frac{\sigma\left(\rho-\rho_{v}\right) g}{\rho_{v}^{2}}\right)^{0.25}
$$

$\mathrm{r}_{\mathrm{lv}}$ is the latent heat of vaporization, $\rho_{\mathrm{v}}$ is the vapor density, $\rho$ is the fluid density, $\sigma$ is the surface tension, and $\mathrm{g}$ is the gravity acceleration.

For locally heated horizontal plates $\mathrm{k}=3.4, \mathrm{n}=-0.5$ [3], for horizontal cylinders $\mathrm{k}=1.4$ [8], and for spheres $\mathrm{k}=2.5$ [8]. The reason for such significant effect of the size of SSS on qcr is connected with the feature of hydrodynamic situation that occurs around the surface of small size (SSS).

The aim of the present work is studing of pool boiling of water on the surfaces with different geometry.

\section{Experimental setup}

The drawing of the working section is shown in Fig.1. The working chamber is formed by a radiator, a steam tube, a cover and a core body. Assembling, compression and sealing is performed by the means of fastening elements - flanges, studs and nuts. Before assembling, a cover is installed on the radiator. The casing consists of a cylindrical shell and two rings. The radiator has the compact form of a multilobal drum with a large heat exchange surface.

For evacuation of air, supply of liquid and installation of a thermocouple, an inclined branch with a union is glued into the working cavity to the cover. Through the cover, it is possible to visually observe the processes occurring in the layer of boiling liquid.

The core assembly is removable and consists of a core, core rings and nozzles. The core is firmly inserted into the ring, and the nozzle is mounted on the end of the core. The core assembly is firmly inserted into the core body. The tightness of the core assembly relative to the housing is provided by a rubber ring that is installed in the outer groove of the core ring. Cover of the core and screw are used for pressing the core to the end of the case. The cover is screwed to the core housing. The screw is screwed into the cover and presses the core assembly.

Two thermocouples are mounted on the core. One thermocouple measures the temperature of the core in the area of contact with the boiling liquid, another thermocouple measures the temperature at the base of the core. In the ring of the core there is a radial groove for laying the wires of the thermocouple. The wires of both thermocouples are discharged through the side openings located in the core body.

When the heat flux is applied to the core, the liquid is heated and boiled in a layer covering the upper surface of the core with a nozzle. The vapor of the liquid passes through the steam pipe and enters the upper part of the radiator. In the radiator, the steam condenses by cooling the outside walls with air. Condensate flows down and flows through the slots of the steam tube into the layer of boiling liquid. For vacuuming, two-stage rotary vane vacuum pump VEB MLW 2DS1 is used. To circulate the liquid, a COLE PARMER EW74905-54 dispenser is used. Feed control is performed from the control panel. The transfer of heat to the core is due to the passage of current through the heating element - a wire wound around the core in a spiral and having a reliable thermal contact with it. The wire is made of a heat resistant material - nichrome, which has a high electrical resistance. The DC power source GW INSTEK GPD-74303S is connected to the ends of the wire. The change in the input power is due to the manual regulation of the voltage at the power supply. To 
monitor the input power in the power supply, indication and recording of current and voltage readings are provided.
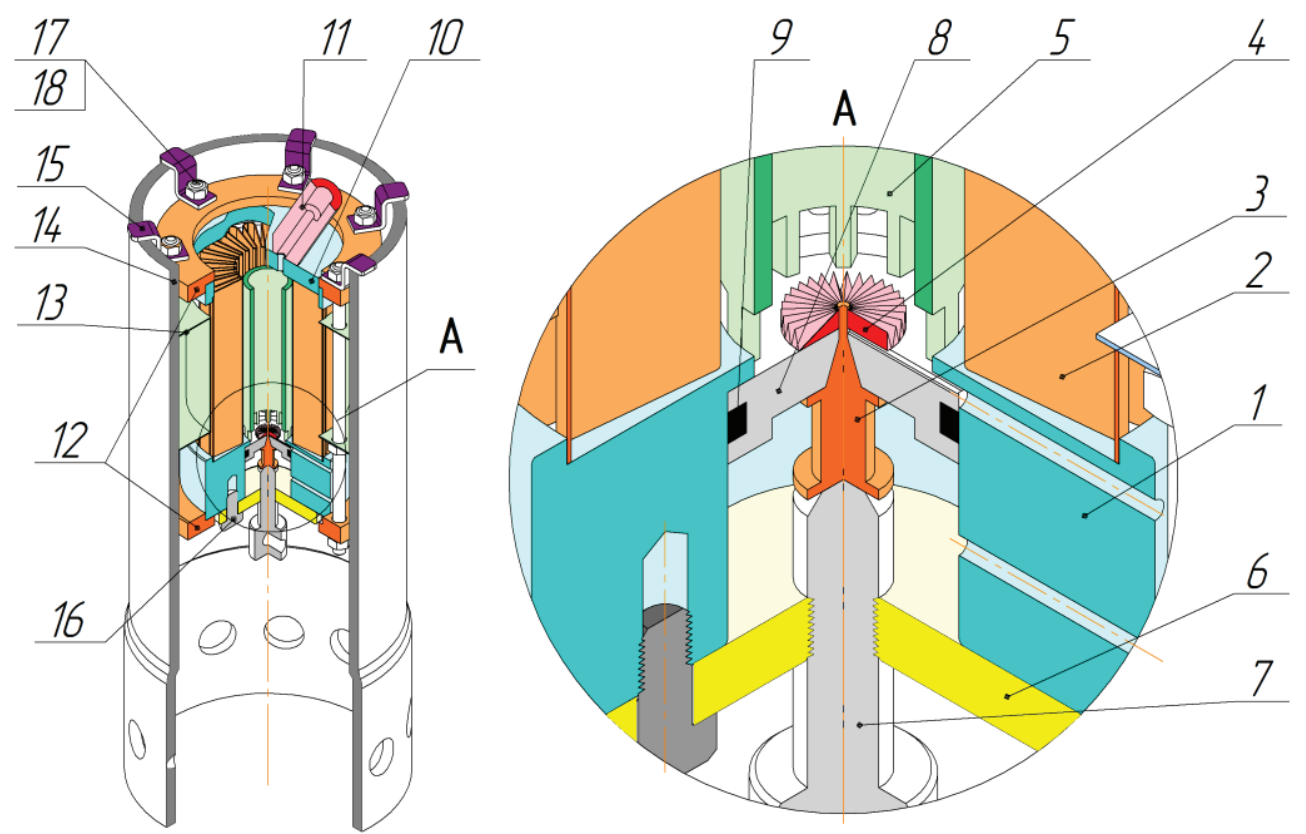

Fig.1 Test section: 1 - core body; 2 - radiator; 3-core; 4 - the core nozzle; 5 - a steam pipe; 6 - the cover of the core; 7 - core screw; 8 - core ring; 9 - a rubber ring; 10 - cover; 11 - withdrawal; 12 flange; 13 - a casing of a radiator; 14 - blowing pipe; 15 - the bracket; 16-screw; 17, 18 - a hairpin and a nut.

Temperature measurement is performed using $\mathrm{K}$ type thermocouples. The thermocouples are connected to the NI TB 9214 measuring module via a 16-channel cDAQ 9171 switching unit. The measuring module is connected to a personal computer (PC) via a USB cable. The PC is equipped with the appropriate software, which allows you to continuously record the values of the corresponding temperatures, build graphical dependencies and archive data. The system uses an analogue absolute pressure sensor WIKA P-30 to measure the vapor pressure above the bed of boiling liquid. The sensor has a measuring range from 0 to 1.6 bar. The output signal of the sensor is $0-10 \mathrm{~V}$. The pressure sensor through the power supply TRACO POWER TXL 750-24S is connected to one of the inputs of the analog measuring module NI USB 6001, which is connected to the PC via a USB cable. The measuring module has 4 analog inputs. The corresponding software is installed on the PC, allowing to record the vapor pressure.

\section{Results and conclusions}

Heat transfer at boiling on local heaters with a diameter of 5 and $10 \mathrm{~mm}$ at the saturation temperature under conditions of a large volume of liquid was studied. It is shown that on the finned surfaces the overheating with respect to the saturation temperature in comparison with a smooth surface decreases to 1.5 times for a heater $10 \mathrm{~mm}$ in diameter and up to three times for a heater with a diameter of $5 \mathrm{~mm}$. Up to two times the coefficient of heat transfer on the finned surface increases in comparison with the smooth one. For finned tips on a 1 $\mathrm{mm}$ diameter heater, surface overheating with respect to the saturation temperature is 
reduced to four times. The coefficient of heat transfer on the finned surface increases by more than three times in comparison with the smooth one.

A comparison of the results obtained for the heat transfer crisis with the literature data for small-sized surfaces is made. It is shown that the data for relatively large diameter heaters coincide with the calculated ones, and for the $1 \mathrm{~mm}$ diameter heater lie substantially higher. The effect of underheating of the liquid to the saturation temperature on the characteristics of the cooling system is studied. It is shown that when the temperature of the liquid surrounding the heat exchange surface decreases, the temperature of the LED crystal model also decreases.

As a result of the experimental work, it is established that heat flux densities in the system can be removed up to $2500 \mathrm{~W} / \mathrm{cm}^{2}$. Moreover, the optimal cooling when the LED semiconductor crystal is heated to a temperature not exceeding $120{ }^{\circ} \mathrm{C}$ is possible for heat flow densities up to $1000 \mathrm{~W} / \mathrm{cm}^{2}$. The expediency of using radial fins with a diameter of 1 $\mathrm{mm}$ is shown. It is recommended to maintain a level of filling the liquid above the heated surface in a thermosyphon of at least $10 \mathrm{~mm}$.

The work was supported by the RF Ministry of Education and Science (Agreement No. 14.604.21.0053, project ID RFMEFI60414X0053).

\section{References}

1. Moor F. D., Mesler R. B. AIChE Journal 7 (1961)

2. Borisov V. Z., Kirillov P. L. Journal of Engineering Physics and Thermophysics 28, 910 (1970)

3. Tolubinskiy, V.I., Antonenko, V.A., Kudritskiy, G.R., Ostrovskiy, Yu.N Industrial Heat Engineering 9 (1987)

4. Chinnov E. A. Journal of Heat Transfer 138 (2016)

5. Kabov O.A., Zaitsev D.V., Cheverda V.V. and Bar-Cohen A. Experimental Thermal and Fluid Science 35, 825 (2011)

6. Moor F. D., Mesler R. B. AIChE Journal 7 (1961)

7. Chinnov E.A., Dyatlov A.V., Kravchenko D.N. Siberian Physico-Technical Journal 5 (1992)

8. Linard D., Dir B. Teploperedacha C 2 (1973).

9. Yu. V. Lyulin, S. E. Spesivtsev, I. V. Marchuk and O. A. Kabov. Technical Physics Letters 41 (2015) 